\title{
La motiline, une hormone intestinale active en période de jeûne
}

La motiline est une hormone peptidique digestive de 22 acides aminés dont on trouve également de faibles quantités dans le système nerveux central. Elle intervient dans l'activité motrice cyclique du muscle intestinal dans les périodes de jeûne et aussi, probablement, dans la stimulation, durant ces périodes, de la sécrétion pancréatique externe.

\section{Pierre Poitras}

Chercheur agrégé à l'université de Montréal

\section{RÉFÉRENCES}

1. Brown JC, Cook MA, Dryburgh JH. Motilin a gastric motor activity stimulating polypeptide : aminoacid sequence. Can J Biochem 1973 ; 51 : 533-7.

2. Poitras P, Reeve JR, Hunkapiller MW, Hood LE, Walsh JH. Purification and characterization of canine intestinal motilin Regul Pept 1983 ; 5 : 197-208.

3. Poitras P, Trudel L, Lahaie RG, PomierLayrargue G. Motilin-like-immunoreactivity in intestine and brain of dog. Life Sci 1987 ; 40 : 1391-5.

\section{ADRESSE}

P. Poitras: centre de recherche clinique André-Viallet hôpital Saint-Luc 1058, rue Saint-Denis, Montréal (Québec) H2X 3J4,

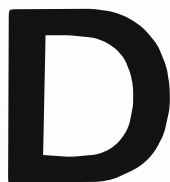

e la muqueuse intestinale porcine, Brown et al. ont isolé en 1973 un polypeptide linéaire de 22 acides aminés, ayant un poids moléculaire de $2,7 \mathrm{kDa}^{*}$ et stimulant la contraction musculaire gastrique, polypeptide qu'ils nommèrent motiline [1]. Ce peptide est identifiable chez plusieurs espèces animales, dont l'homme, mais c'est chez le chien qu'a été accomplie la majorité des travaux qui ont, entre autres, démontré son implication dans le contrôle de la motricité gastro-intestinale et de la sécrétion exocrine du pancréas.

\section{Structure moléculaire}

La motiline ne présente aucune similitude structurale avec d'autres peptides actuellement purifiés dans une quelconque espèce animale. La motiline canine est structurellement

${ }^{*} k D a=$ kilodalton. différente de la motiline porcine [2] ; comme le démontre la figure 1, cinq acides aminés y sont substitués dans la partie centrale de la molécule. Cette hétérogénéité structurale d'espèce entraînera des caractéristiques fonctionnelles et immunologiques particulières aux motilines de ces deux espèces, ainsi que nous en parlerons plus loin. Une hétérogénéité moléculaire est aussi retrouvée. Chez le chien, la forme moléculaire de 22 acides aminés est nettement prédominante tant dans' les tissus que dans le sang circulant. Lors de chromatographies sur tamis moléculaire (G50 Séphadex) d'extraits intestinaux, on pourra suspecter la présence, en faible quantité toutefois, de deux «promotilines » [3]. La motiline humaine est identique à la motiline porcine, ainsi que nous l'a révélé tout récemment la séquence partielle de l'ADNc de motiline humaine (Dea, Lahaie, Poitras, observation non publiée). La présence, tout comme chez le chien, de précurseurs 


$$
\text { -HIS-SER- }
$$

-LYS-ISO-ARG-

Phe-VAL-Pro-ILE-Phe-Thr-TYr-GLY-GLU-LeU-GLN-ARG-Met-GLN-

$$
78
$$

GLU-LYS-GLU-ARG-ASN-LYS-GLY-GLN

Figure 1. Séquence des acides aminés composant la motiline porcine. Les substitutions propres à la motiline canine sont indiquées au-dessus des acides

\begin{tabular}{|c|c|c|c|c|}
\hline Duodénum & Muq & & $100 \%$ & \\
\hline Jéjunum & Muq & $65 \%$ & $0,5 \%$ & Pinéale \\
\hline Duodénum & Mus & $38 \%$ & $1,7 \%$ & Noyau caudé \\
\hline Jéjunum & Mus & $\square 31 \%$ & $2,4 \%$ & Cortex (frontal) \\
\hline lléon & Muq & $14 \%$ & $2,8 \%$ & Bulbe \\
\hline lléon & Mus & $4 \%$ & $2,8 \%$ & Thalamus \\
\hline Côlon & Muq & $2,5 \%$ & $3,2 \%$ & Cervelet \\
\hline Antre & Muq & $1,5 \%$ & $3,6 \%$ & Noyau strié \\
\hline Fundus & Muq & $1,5 \%$ & $3,7 \%$ & Moelle \\
\hline Côlon & Mus & $0,8 \%$ & $4,1 \%$ & Hypothalamus \\
\hline Antre & Mus & $0,75 \%$ & $10,3 \%$ & Hypophyse ant. \\
\hline Fundus & Mus & $0,5 \%$ & $13,0 \%$ & Hypophyse post. \\
\hline
\end{tabular}
aminés occupant les positions 7-8-12-13-14.

Figure 2. Distribution de la motiline dans diverses régions du tube digestif (à gauche) ou du cerveau (à droite). Les valeurs indiquées pour chaque région représentent le pourcentage de la concentration maximale retrouvée dans la muqueuse duodénale. Mus : musculeuse; Muq: muqueuse; ant : antérieure ; post : postérieure. (D'après [3]).

d'environ 14 et 6 kDa y est suspectée. Cependant, la proportion de l'immunoréactivité totale attribuable à ces «promotilines», tant dans les tissus que dans la circulation périphérique, apparaît plus importante chez l'homme que chez le chien et, surtout, variable selon les antisera utilisés pour les détecter. La séquence et l'activité biologique du (des) précurseur(s) sont inconnues.

$\mathrm{m} / \mathrm{s} n^{\circ} 5$ vol. 4, mai 88 ponsable de la synthèse de la motiline demeure encore sujet de discorde; selon l'anticorps (spécifique pour la portion $\mathbf{N}$ ou $\mathbf{C}$ terminale) utilisé pour la détection immunohistochimique, on pourra retrouver la motiline dans les cellules entérochromaffines ou encore dans d'autres cellules non entérochromaffines. La controverse concernant la possibilité que ces résultats reflètent la présence de formes moléculaires multiples de motiline ou de peptides différents mais appartenant à une même famille peptidique, n'a toujours pas été résolue [5].

\section{Relation}

\section{structure-fonction}

La motiline porcine entraîne, in vitro, une contraction du muscle duodénal du lapin et de l'homme [6]. Cette contraction, proportionnelle à la dose utilisée, est insensible à de nombreux bloquants, dont l'atropine et la tétrodotoxine (inhibiteur de la conduction axonale), suggérant donc un effet direct du peptide sur la cellule musculaire de ces espèces animales. Des fragments synthétiques contenant les 16 premiers ou les 16 derniers acides aminés de la motiline porcine semblent dénués d'activité biologique. Le remplacement du premier acide aminé de la séquence entraîne, à lui seul, une diminution importante de l'activité biologique de la molécule et est donc probablement nécessaire à l'expression de son activité biologique. L'hétérogénéité d'espèces concernant la structure des motilines canine et porcine (et humaine) entraîne toutefois des conséquences biologiques. Nos expériences in vitro [7] ont démontré que le muscle duodénal canin possédait un récepteur spécifique à la motiline canine, insensible à la motiline porcine (figure 3, page 300). Ces deux motilines apparaissent toutefois également actives in vivo. Même si, in vitro, la motiline canine a une action directe (non bloquée par la tétrodotoxine) sur le muscle lisse intestinal, son action chez le chien in vivo, tout comme celle de la motiline porcine [8], est inhibée par l'atropine, l'hexaméthonium (bloqueur nicotinique ganglionnaire) ou la tétrodotoxine, démontrant donc une parti- 
4. Chey WY, Escoffery R, Roth F, Chang TM Yajima $\mathrm{H}$. Motilin-like-immunoreactivity in the gut and neurones of peripheral and central nervous system. Gastroenterology 1980 ; $78: 1150$.

5. Polak JM, Buchan AMG. Motilin immunocytochemical localization indicates possible molecular heterogeneity or the existence of a motilin family. Gastroenterology 1979; $76: 1065$.

6. Strunz U, Domschke W, Mitznegg P, et al. Analysis of the motor effects of 13-nor-leucine motilin on the rabbit, guinea pig, rat and human alimentary tract in vitro. Gastroente rology 1975 ; 68 : 1485-91.

7. Poitras P, Lahaie RG, St-Pierre S, Trudel L. Comparative stimulation of motilin duodenal receptor by porcine or canine motilin. Gastroenterology 1987 ; $92: 658-62$.

8. Fox JET, Daniel EE, Duory J, Robothan E. The mechanisms of motilin excitation of the canine small intestine. Life Sci $1984 ; 34$ : $1001-6$.

9. Lee KY, Shiratori K, Chen YF, Chang TM, Chey WY. A hormonal mechanism for the interdigestive pancreatic secretion in dogs Am J Physiol 1986 ; 14: G759-64.

10. Magee DF, Naruse S. The role of motilin in periodic interdigestive pancreatic secretion in dogs. J Physiol (Lond) 1984 ; 355 : 441-7.

11. Code CF, Marlett JA. The interdigestive myoelectric complex of the stomach and small bowel of dogs. J Physiol (Lond) 1975 ; 246 : 289-309.

12. Vantrappen G, Janssens J, Hellemans J, Ghoos Y. The interdigestive myoelectric complex in patients with bacterial overgrowth of the small intestine. J Clin Invest 1977; 59 : 1158-68.

13. Thomas PA, Kelly KA. Hormonal control of interdigestive motor cycles of canine proximal stomach. Am J Physiol $1979 ; 237$ : 192-7.

14. Poitras P. Motilin is a digestive hormone in dog. Gastroenterology $1984 ; 87$ : 909-13.

15. Lee KY, Chang TM, Chey WY. Effect of rabbit anti-motilin serum on myoelectric activity and plasma motilin concentration in fasting dogs. Am J Physiol 1983 ; 245 : G54753.

16. Janssens J, Vantrappen G, Peeters TL. The activity front of the migrating motor complex of the human stomach but not of the small intestine is motilin- dependant. Regul Pept $1983 ; 6: 363-9$.

17. Phillis JW, Kirkpatrick JR. Motilin excites neurones in the cerebral cortex and spinal cord. Eur J Pharmacol 1979; 58: 46972

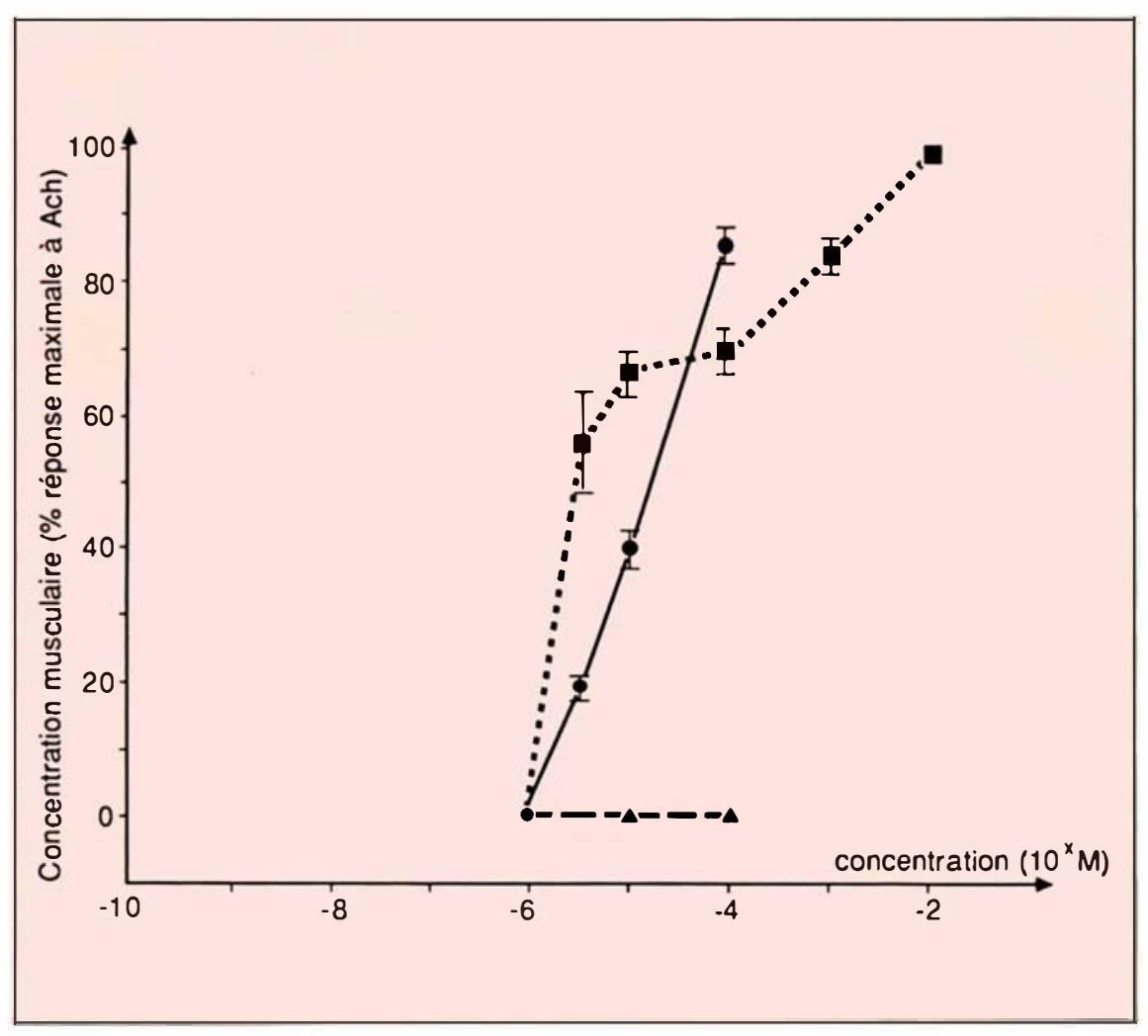

Figure 3. Contraction du muscle duodénal canin lors de stimulations in

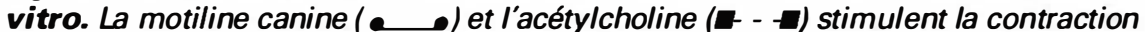
musculaire alors que des doses similaires de motiline porcine $(\Delta--\Delta)$ demeurent sans effet. (D'après [7]).

cipation essentielle du système nerveux intrinsèque cholinergique à l'expression de l'activité biologique (Poitras, travaux non publiés). Les données actuelles suggèrent donc que des récepteurs de la motiline sont situés à deux endroits distincts au niveau de l'intestin canin: le muscle intestinal, mis en évidence in vitro, et les nerfs stimulateurs cholinergiques. Ces derniers récepteurs insensibles à l'hétérogénéité structurale d'espèce sont révélés par les expériences in vivo. A ce jour, on suspecte que, chez l'animal conscient, le récepteur nerveux soit doté d'une plus grande affinité pour le peptide et exerce donc une activité biologique prépondérante. Dans d'autres espèces animales, incluant l'homme, les études pour identifier le mode d'action in vivo de la motiline sont toujours à faire.

La motiline peut aussi stimuler la sécrétion exocrine du pancréas chez le chien [9] ; cette action semble, elle aussi, nécessiter la participation d'un médiateur cholinergique $[9,10]$.

\section{Activité physiologique}

La recherche du rôle physiologique de la motiline a donné lieu à l'exploration concomitante de l'activité interdigestive, une fonction encore mal connue jusqu'à tout récemment. Plusieurs organes digestifs, tant moteurs que sécrétoires, que l'on croyait encore tout récemment au repos entre les repas et stimulés uniquement à la suite d'une ingestion alimentaire, sont aussi actifs en période de jeûne (période interdigestive). C'est au début des années 1970 qu'a été reconnue l'existence, au niveau de activité motrice intermittente mais régulière survenant durant le jeûne, et appelée le «complexe migrant interdigestif [11]; cette activité $\mathrm{m} / \mathrm{s} n^{\circ} 5$ vol. 4 , mai 88 l'estomac et de l'intestin grêle, d'une 


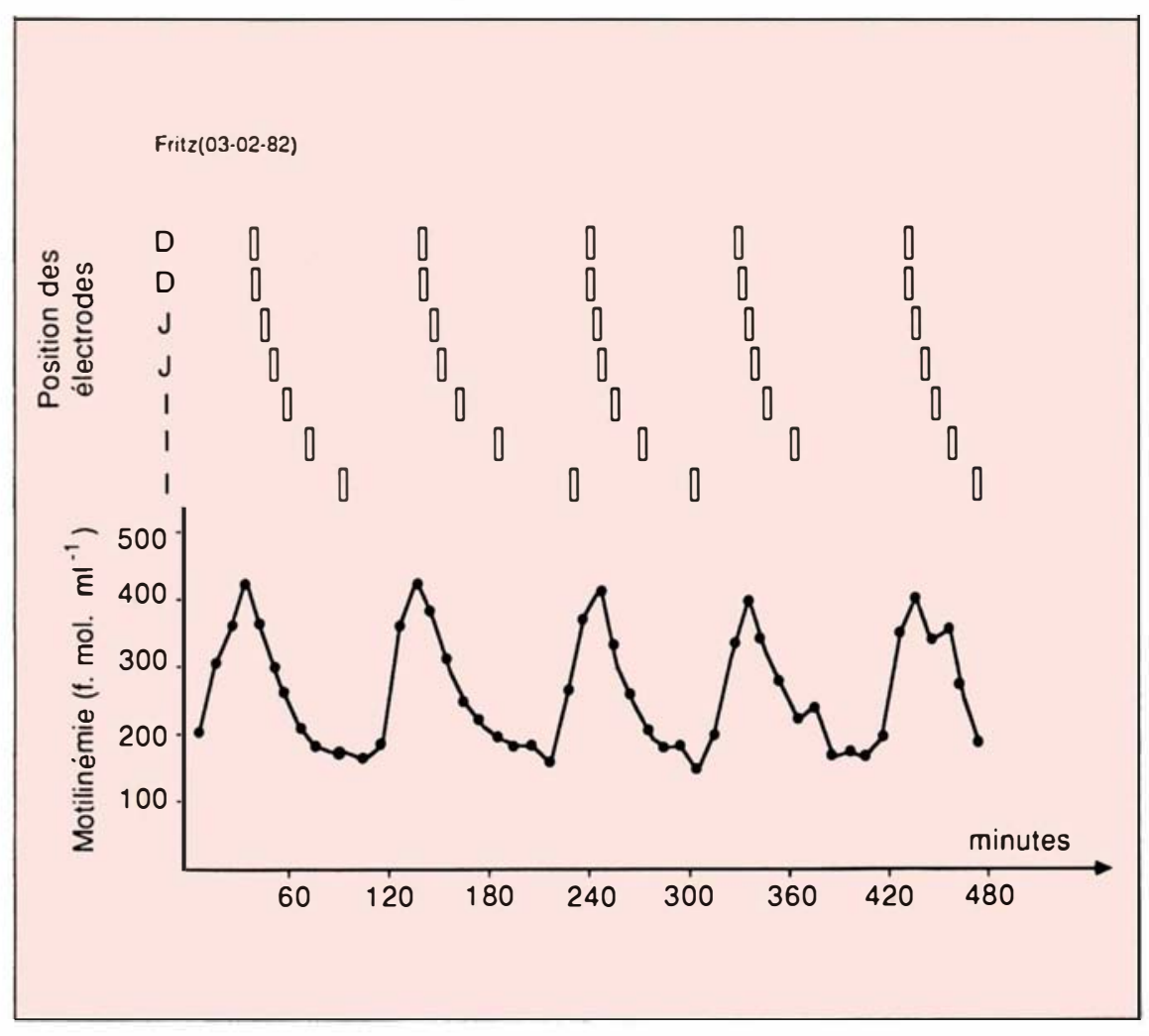

Figure 4. Fluctuations de la motilinémie lors de la période interdigestive chez un chien, coïncidant avec les périodes de phase III (indiquées par les rectangles gris dans la moitié supérieure de la figure) migrant du duodénum (D) au jéjunum (J) et à I'iléon (I). (D'après [25]).

constitue la structure organisationnelle basale de la motricité du tube digestif. Cette entité cyclique et récurrente, d'une durée d'environ $90 \mathrm{~min}$, se compose de quatre phases: La phase I, s'étendant de 20 à 40 minutes et caractérisée par une inactivité musculaire presque totale, sera suivie de la phase II, durant, de 20 à 40 minutes, où l'on constatera une activité motrice modérée et irrégulière de la paroi intestinale. La phase III, ne durant que cinq à dix minutes, sera l'activité motrice la plus caractéristique du complexe migrant interdigestif. Durant cette période, un ensemble de puissantes contractions surviendra et migrera, en 90 minutes environ, le long du tube digestif, du sphincter œsophagien inférieur jusqu'à l'iléon terminal. Suite à ce mouvement lentement propagé, l'activité intestinale décroîtra rapidement (phase IV, durant de cinq à dix minutes) jusqu'à une $\mathrm{m} / \mathrm{s} n^{\circ} 5$ vol. 4, mai 88 et/ou III du complexe migrant interdigestif. L'importance physiologique de ces phénomènes moteurs et sécrétoires se produisant durant la période interdigestive est encore incertaine. On pense que ces phénomènes pourraient servir à «nettoyer le tube digestif » des bactéries et particules alimentaires pouvant s'y accumuler entre les repas ou incomplètement évacuées par l'activité motrice post-prandiale. Ce concept théorique, élaboré par C.F. Code en 1974, semble trouver appui dans certaines observations cliniques; en effet, chez des patients atteints de pullulation bactérienne intestinale d'origine indéterminée, on a constaté une absence de phase III, suggérant ainsi que ce déficit moteur puisse être la cause de la pullulation bactérienne et de la malabsorption ainsi engendrée [12].

Les études menées par Thomas et Kelly [13] chez des chiens ayant subi une dénervation et une auto-transplantation gastrique ou jéjunale, ont bien démontré que l'activité motrice interdigestive de l'estomac (et non du jéjunum) était influencée par des médiateurs présents dans la circulation sanguine. Nos expériences [14] ont clairement identifié le rôle hormonal de la motiline dans le contrôle du complexe migrant interdigestif : 1) l'administration par voie exogène de motiline canine, reproduisant des concentrations sanguines physiologiques, induit une activité motrice comparable à la phase III du complexe migrant interdigestif ; 2) en période de jeûne, les taux de motilinémie sont soumis à des fluctuations continuelles dont les augmentations correspondent parfaitement à l'apparition des phases III (figure 4). Cependant, compte tenu de l'hétérogénéité structurale d'espèce, la motilinémie chez le chien ne sera évaluée correctement que par des anticorps spécifiques de la région $\mathrm{C}$ terminale de la molécule. De plus, il faut ici préciser que la motiline semble responsable de l'induction des phases III exclusivement d'origine gastro-duodénale; des phase III, dites ectopiques, pourront être initiées dans des régions plus distales du tube digestif, sans l'aide de la motiline et probablement via un processus non hormonal, tel que cela est suggéré par les travaux de Thomas 


\section{RÉFÉRENCES}

18. Samson WK, Lumpkin MD, McCann SM. Motilin stimulates growth hormone release in vitro. Brain Res Bull 1982; $8: 117$ 21.

19. Olson RD, Kastin AJ, VonAlmen TK Coy DH, Olson GA. Systemic injections of gastrointestinal peptides alter behaviour in rats. Peptides $1980 ; 1: 383-5$.

20. Porreca F, Dray A. Motilin acts within the CNS to inhibit urinary bladder contractions. Life Sci 1984 ; 34 : 2577-81.

21. Hashamoni M, Go VLW, Taksh T, Szurszewski JH. Effect of central administration of motilin on migrating complexes in the dog Am J Physiol 1987 ; 252 : G195-9.

22. Diamant NE. Neurological control of the interdigestive migrating complex. In : Poitras $\mathrm{P}$, ed. Small Intestinal and Colonic Motility. Proceedings of the Ith International Symposium. Montréal : Laboratoires Jouveinal, $1985 ; 3-13$.

23. Lemoyne $\mathbf{M}$, Wassef $\mathbf{R}$, Tassé $\mathrm{D}$, Trude $\mathrm{L}$, Poitras P. Motilin and the vagus in dog. Can J Physiol Pharmacol 1984 ; 62 : 1092-6.

24. Telford GL, Go VLW, Szurszewski JH. Effect of central sympatectomy on gastric and small intestinal myoelectric activity and plasma motilin concentration in the dog. Gastroenterology 1985 ; 89 : 989-95.

25. Poitras P. Hormonal control of the IDMC. In : Poitras P, ed. Small Intestinal and Colonic Motility. Proceeding of the Ith International Symposium. Montréal : Laboratoires Jouveinal, 1985 ; 15-23.

26. Itoh Z, Nakaya M, Suzuki T, Arai H, Wakabayashi K. Erythromycin mimics exogenous motilin on gastrointestinal contractile activity in the dog. Am J Physiol $1984 ; 247$ G688-94.

27. Hall KE, Greenberg GR, El Sharkawy TY, Diamant NE. Relationship between porcine motilin-induced migrating motor complex-like-activity, vagal integrity, and endogenous motilin release in dogs. Gastroenterology 1984 ; 87 : 6-85.

28. Poitras P, Steinbach JH, Vandeventer G, Code CF, Walsh JH. Motilin independen estopic fronts of the IDMC. Am J Physiol 1980 ; 239 : G215-20.

29. Noseda A, Peeters TL, Delhaye M, Bormans V, Vandermoten G, Yernault JC. Is plasma motilin a tumor marker for small cell carcinoma of the lung ? Can J Physiol Phar macol 1986; (suppl.): 144 (abstr.).

30. Labo G, Bertolotti M, Vezzadini P, Bonora G, Bersani G. Interdigestive gastroduodenal motility and serum motilin levels in patients with idiopathic delay in gastric et Kelly[13] ; 3) finalement, l'administration intraveineuse d'un immunsérum spécifique de la motiline entraîne une disparition totale des phases III dans la région gastroduodénale. Des résultats tout à fait similaires ont été obtenus par Lee $e t$ al. [15]. A la lumière de ces données, on peut certainement conclure au rôle physiologique de la motiline dans le contrôle de la motricité intestinale interdigestive chez le chien. De façon tout aussi convaincante, Lee $e t$ al. [9] ont récemment démontré le contrôle physiologique de la sécrétion exocrine du pancréas par la motiline circulante durant la période interdigestive chez le chien. Ils ont en effet observé: (1) qu'il existait une corrélation parfaite entre l'augmentation cyclique de la sécrétion pancréatique exocrine (volume, bicarbonate, trypsine), de la motricité gastrointestinale et de la motilinémie; (2) que l'administration de quantités physiologiques de motiline exogène entraînait une stimulation de la sécrétion pancréatique; (3) que la perfusion d'antisérum antimotiline abolissait complètement les augmentations cycliques de cette sécrétion. La motiline, chez le chien, est donc une hormone, c'est-à-dire une substance qui, par la variation de ses taux sériques, contrôle des phénomènes physiologiques tels l'induction des phases III du complexe migrant interdigestif au niveau de la région gastro-duodénale et la sécrétion pancréatique exocrine lors de la période de jeûne. Il apparaît que d'autres peptides circulants, dont le polypeptide pancréatique ou la gastrine, sont eux aussi soumis à des variations durant la phase interdigestive; cependant, l'importance physiologique de tous ces phénomènes reste à préciser.

Le complexe migrant interdigestif peut aussi être identifié chez l'homme. Tout comme chez le chien, on suspecte que la motiline puisse y être responsable de l'organisation phasique de la motricité gastro-intestinale interdigestive. Cependant, la démonstration définitive de son implication physiologique reste à faire. Des écueils techniques, concernant principalement la validation d'un dosage radioimmunologique, évaluant de façon adéquate la moti-

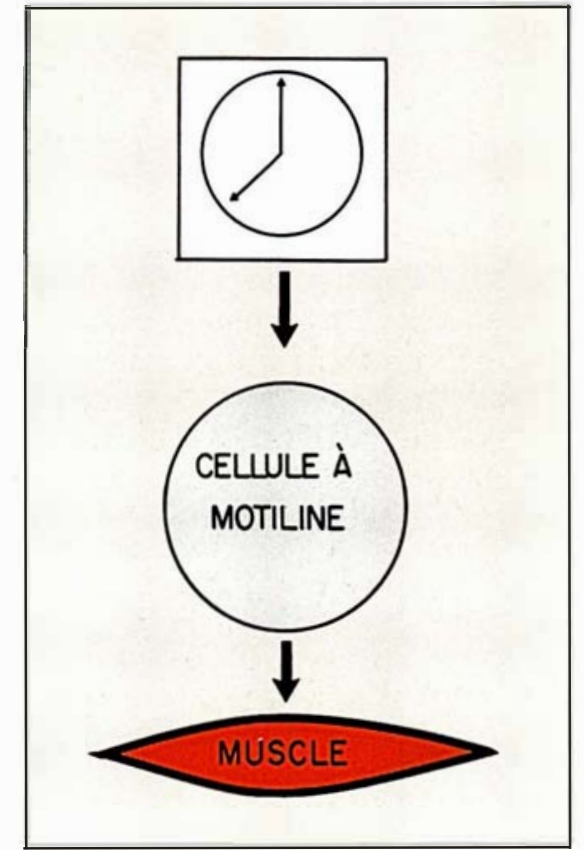

Figure 5. Schéma représentant, de facon hypothétique, la stimulation du muscle intestinal par la motiline dont la sécrétion cyclique est contrôlée par un mécanisme d'origine et de nature toujours inconnues.

linémie chez l'humain et l'enregistrement précis de la motricité gastrointestinale, doivent encore être surmontés. Cependant, compte tenu de la capacité de la motiline exogène à induire des phases III chez l'homme et des études suggérant une certaine corrélation entre les variations de motilinémie et de motricité digestive [16], il est plausible que, tout comme chez le chien, la motiline soit un médiateur physiologique de la motricité gastro-intestinale chez l'homme.

L'importance physiologique de la motiline en tant que neurotransmetteur est inconnue. On retrouve la motiline au niveau du système nerveux central (figure 2). Des travaux récents ont démontré que la motiline était un puissant excitateur de neurones de la moelle épinière d'amphibien [117], qu'elle pouvait stimuler la sécrétion hypophysaire d'hormone de croissance chez le rat [18], et accroître l'impression de satiété chez 
ce même animal [19]. De plus, l'administration intracérébroventriculaire de motiline peut inhiber le réflexe de miction chez le rat [20], mais est incapable de stimuler la motilité intestinale chez le chien [21].

\section{Contrôle de la sécrétion}

La motiline est donc un stimulus essentiel (mais non nécessairement unique) au contrôle du complexe migrant interdigestif. On imagine schématiquement (figure 5) qu'il existe une «horloge» gérant les variations cycliques de motilinémie, variations qui influenceront (en collaboration probable avec d'autres facteurs) la contraction du muscle lisse intestinal (et la sécrétion pancréatique). L'emplacement et la nature de cette horloge sont inconnus. Il est démontré que le système neurologique intrinsèque de l'intestin a un rôle essentiel à jouer dans l'initiation et la fréquence intrinsèque du complexe migrant interdigestif [22]. L'innervation extrinsèque et les substances hormonales (dont la motiline) peuvent probablement influencer le mécanisme neurologique intrinsèque. Nous avons observé que la stimulation du système nerveux central à l'aide d'un repas "fantôme» pouvait chez le chien inhiber la sécrétion de motiline et le développement de la phase III [23]. Le cycle de sécrétion de la motiline et la motricité intestinale ne sont pas altérés significativement chez l'animal ayant subi soit une vagotomie [23], soit une sympathectomie abdominale, ou une section médullaire haute [24].

Il est actuellement impossible de présenter un schéma global des mécanismes physiologiques régissant les variations de la motilinémie. Certains agents peuvent stimuler une libération de motiline chez le chien [25], où la plupart des études ont été réalisées. L'injection intraduodénale d'une solution alcaline ou de sels biliaires entraîne une augmentation de la motilinémie. L'administration intraveineuse d'acétylcholine, de bombésine, d'opiacés ou d'antibiotiques de type érythromycine [26] a une action similaire. Il a même été rapporté que l'injection de motiline exogène entraîne une libération de motiline endogène [27], suggérant un processus de rétroaction stimulatrice.

L'ingestion d'un repas inhibe la motricité intestinale interdigestive et la sécrétion cyclique de motiline [28]. Des mécanismes hormonaux semblent impliqués dans ce passage de la motricité interdigestive vers une motricité de type post-prandial[13] sans que les médiateurs physiologiques de cet événement soient connus. Plusieurs substances telles l'atropine, la somatostatine, l'insuline, le polypeptide pancréatique (à dose pharmacologique), et la naloxone peuvent entraîner une diminution de la motilinémie et une inhibition des phases III au niveau du tube digestif supérieur [25]. La gastrine, la cholécystokinine, la sécrétine et le polypeptide pancréatique (à dose physiologique), modifient la motricité gastro-intestinale sans toutefois influencer la sécrétion cyclique de motiline.

\section{La motiline en pathologie humaine}

Il n'a pu être retrouvé, jusqu'à maintenant, de situation pathologique impliquant précisément la motiline comme processus étiopathogénique. Les connaissances physiologiques actuelles suggèrent qu'une hypersécrétion de motiline pourrait engendrer une hyperactivité motrice intestinale, alors que, à l'opposé, un déficit de motiline s'accompagnerait d'une hypoactivité. Aucun cas de motilinome n'a jamais été rapporté, même si Dive (communication personnelle) a observé une malade ayant une diarrhée sévère d'origine indéterminée et chez qui la motilinémie était très élevée. Noseda et al. [29] ont de plus retrouvé une augmentation importante de la motilinémie chez des malades atteints de tumeurs pulmonaires à petites cellules. Par ailleurs, on pourrait suspecter qu'une déficience en motiline puisse jouer un certain rôle dans l'étiopathogénie de certaines atonies affectant l'estomac ou l'intestin grêle. A titre d'exemple, Labo et al. [30] ont identifié chez des malades souffrant de gastroparésie idiopathique un déficit de phase III dans la région gastroduodénale; chez la moitié de ces sujets, on constata une absence d'élévation cyclique de motiline

\section{Summary}

Motilin, a 22 amino acids polypeptide synthetized in endocrine cells of the duodenum and jejunum is found in the peripheral circulation of various animal species. In the dog, motilin plays a physiological endocrine role during the fasting period, regulating the motor function of the stomach and the small intestine (inducing the phase III of the interdigestive migrating complex) and the secretory function of the exocrine pancreas. Motilin is also found in man where a similar role is also suspected but still remains to be confirmed. Interspecies structural heterogeneity and intra-species molecular heterogeneity are present and can influence the bioactive and immunoreactive characteristics of the molecule. Motilin is released cyclically in the blood during the fasting period, and, although numerous substances can influence plasma motilin levels, the mechanisms regulating this cyclic phenomenon are still unclear. Alteration in motilin release could be involved in intestinal motility disorders.

\section{TIRÉS A PART}

P. Poitras: centre de recherche clinique André-Viallet hôpital Saint-Luc 1058, rue Saint-Denis, Montréal (Québec) H2X 3J4, Canada. 